\title{
The periodic structure topology optimization using improved genetic algorithm
}

\author{
Qu Dongyue ${ }^{1, a}$, Huang Yangyang ${ }^{2, b}$ and Song Jinyu ${ }^{3, c}$ \\ ${ }^{1}$ School of Harbin, engineering University, Harbin 150000, China; \\ ${ }^{2}$ School of Harbin, engineering University, Harbin 150000, China; \\ ${ }^{3}$ School of Northeast, agricultural University, Harbin 150000, China. \\ aqudongyue@hrbeu.edu.cn, ’huangyangyang2009@163.com, 'brucesong778899@163.com
}

Keywords: Genetic algorithm; Bi-directional evolutionary structural optimization algorithm; Periodic structure optimization

\begin{abstract}
This paper combine improved genetic algorithm with bi-directional evolutionary structural optimization algorithm to optimize structure in dual coding way. The new algorithm not only has solved the genetic algorithm (GA) problem which is easy to appear premature, but also has solved the bi-directional evolutionary structural optimization algorithm (BESO) problem which is easy to get a local optimal solution. In order to satisfy the constraints of the periodic structure, the design area is divided into the form of unit cells, and optimize the representative unit cell, other cells content repeat constraints. This paper optimize the typical periodic structure with the new method of topology optimization, and the optimization results show that the presented method is reliability and validity.
\end{abstract}

\section{Introduction}

Since the BESO method proposed [1], topology optimization of continuum structure has got rapid development. In practical engineering, Because of the lightweight and manufacturing simplicity requirements, periodic structure topology optimization is widely used in aviation, construction and other fields, so it has higher used value and more practical significance to study periodic structure topology optimization. Huang and Xie [2] used the method of BESO to optimize the periodic structure, based on it, this paper proposed the algorithm which is combining improved genetic algorithm with bi-directional evolutionary structural optimization algorithm (IGA-BESO) for topology optimization, which overcame the local optimal solution may occur in the BESO algorithm [3], but also overcome the GA premature problems in the optimization process. Examples prove that the proposed method has the reliability and validity.

\section{The sensitivity analysis}

In order to satisfy the periodic constraint conditions, this paper divide the design area into unit cells according to the number of constraints in all directions, and the whole design area is divided into $\mathrm{m}=\mathrm{m} 1 \times \mathrm{m} 2$ same unit cells, as shown in figure 1 .

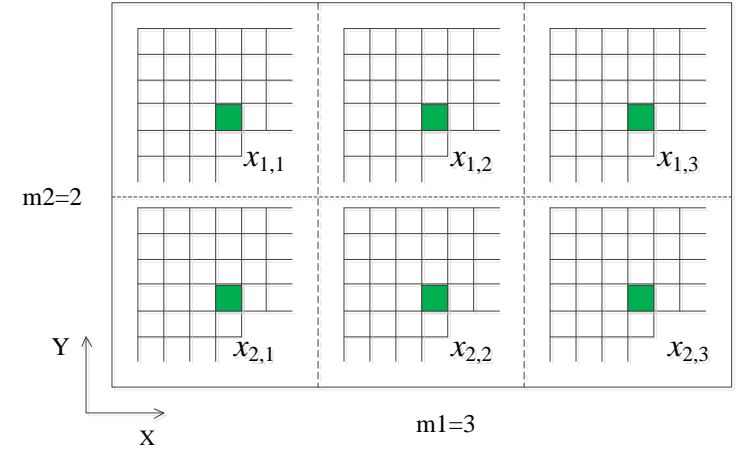

Figure. 1 The diagram of the unit cells in the periodic structure 
This paper use the IGA - BESO method to minimize the structure compliance, and the structure topology optimization model building as shown in the formula (1).

In formula (1), $\mathrm{C}$ is the even compliance of the structure, $\mathrm{F}$ and $\mathrm{U}$ is the load and displacement

$$
\begin{gathered}
\text { Minimize: } C=\frac{1}{2} \mathrm{~F}^{T} U=\frac{1}{2} U^{T} \mathrm{~K} U=\frac{1}{2} \sum_{i=1}^{N} x_{i}^{p} u_{i}^{T} \mathrm{k}_{0} u_{i} \\
\text { Subject to: } \mathrm{V}^{*}-\sum_{i=1}^{m} \sum_{j=1}^{n} \mathrm{~V}_{i, j} x_{i, j}=0 \\
x_{1, j}=x_{2, j}=x_{3, j}=\ldots=x_{m, j} \\
x_{i, j}=x_{\min } \text { or } 1
\end{gathered}
$$

vector in the structure respectively, $\mathrm{V}^{*}$ is the target volume, $\mathrm{V}_{i, j}$ is the volume of the $j$ th element in the $i$ th cell, $x_{i, j}$ is the density values of the $j$ th element in the $i$ th cell.

The periodic structure is optimized by the dual coding way in this paper, but only encode the element in the representative unit cell. On the macroscopic, 0 represent the absence of the element and 1 represent presence. On the microscopic,this paper endows individuals in representative unit cell with a certain string length of gene, according to the sensitivity of the element in representative unit cell, divide the element in different classes, then individual implement crossover and mutation in inter classes to update gene, add or delete material. In order to satisfy the constraints of periodic structure, different unit cell in corresponding location of unit cells should have the same sensitivity value and the density value. according to the formula (1), it can deduce the sensitivity of the elements in the representative unit cell, as shown in the formula (2). The sensitivity of element in representative unit cell should be assigned to other cells elements in corresponding location.

$\alpha_{j}=\frac{d C}{d x_{j}}=\sum_{i=1}^{m} \frac{1}{2} x_{i, j}^{p-1} u_{i, j}^{T} \mathrm{~K}_{i, j} u_{i, j}$

$\alpha_{j}$ is the sensitivity of the jth element in the representative unit cell, $\mathrm{m}$ is the number of the unit cells, $\mathrm{p}$ is the penalty factor, and its general values is more than 2 .

\section{Topology Optimization Process of IGA-BESO}

The topology optimization process of the IGA-BESO method as follow figure 2.

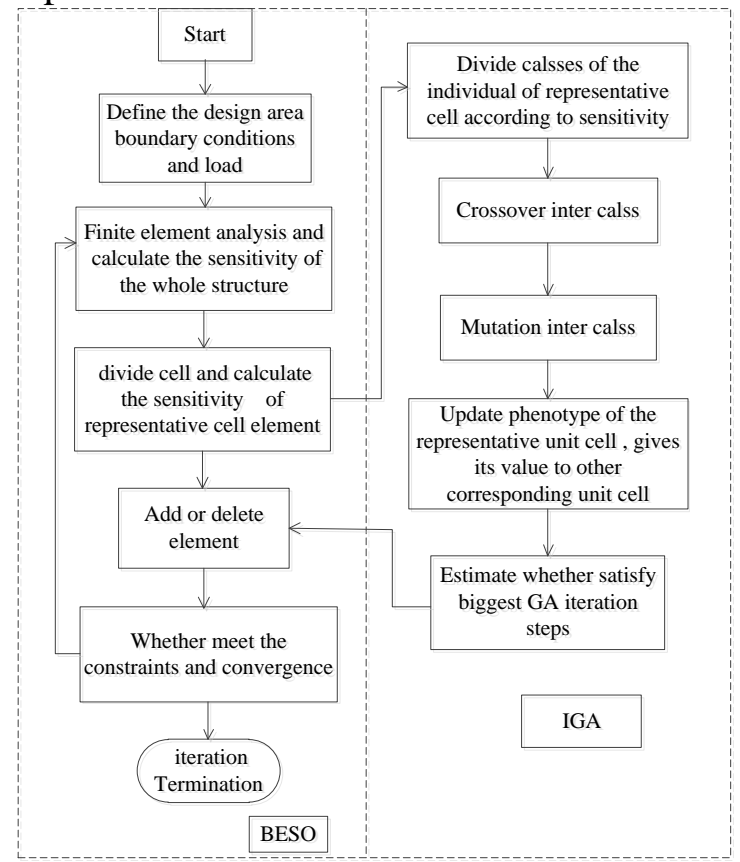

Figure. 2 The topology optimization process of the IGA-BESO method 


\section{Calculation and result analysis}

(1) 2D double side fixed beam

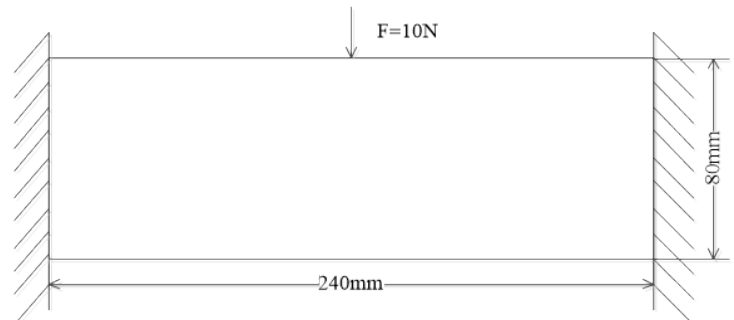

Figure. 3 Load and constraints of the double side fixed beam

As shown in the figure 3 , the design area of the structure is a rectangular with $240 \mathrm{~mm} \times 80 \mathrm{~mm}$, the degree freedom of $\mathrm{X}$ and $\mathrm{Y}$ directions is fixed at the left end and right end of the structure. A vertical concentrated load $\mathrm{F}=10 \mathrm{~N}$ is applied at the center of the up face. The elastic modulus of materials is $100 \mathrm{MPa}$. Poisson's ratio is 0.3 . The volume constraint is 0.75 . The filter radius is $10 \mathrm{~mm}$. Selected different unit cell numbers, optimization topology structure with the IGA-BESO method is shown in the table 1 below.

It can be seen from the table 1, the final optimization topology structure has a big difference when the cell number is different in the structure, and with the increase of the unit cell number, the topology structure is more and more complex. The number of unit cell is equal to the number of periodic constraint. Even compliance and total iteration steps of the two-dimensional double side fixed beam is shown in the following table 2.

As known form the table 2, with the increase of the unit cell number, the even compliance value of the structure is more and more large, because the more number of cell divided, the less element the representative unit cell contains, and it is more difficulty to search the optimal solution in the smaller range area, finally the optimal solution is worse.

Table 1 Optimal designs for 2D double side fixed beam under different constraints

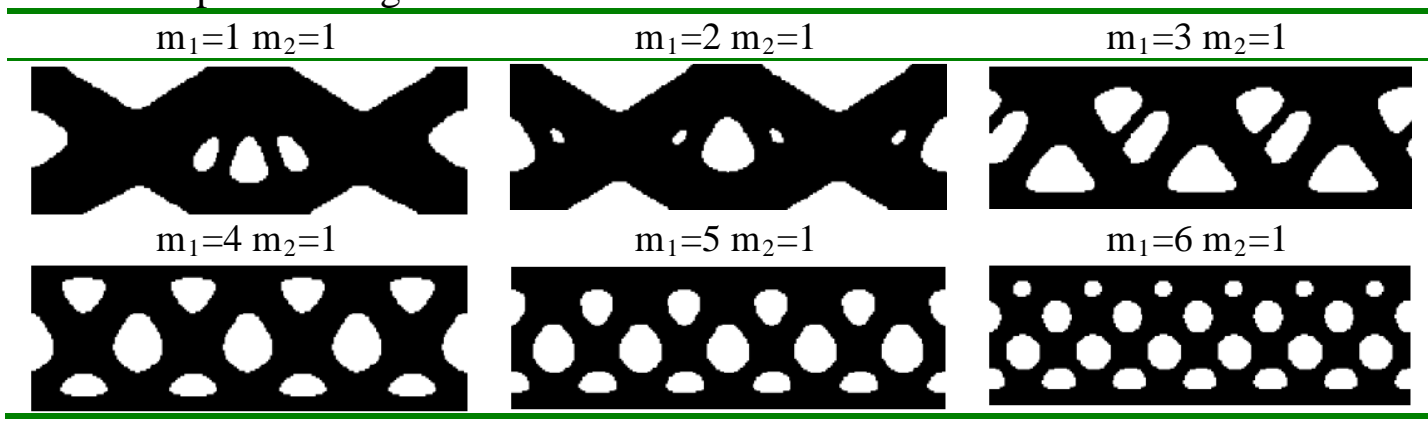

The even compliance and the total iteration steps of the two-dimensional double side fixed beam is shown in the following table 2.

Table 2 The even compliance and the total iteration steps of the double side fixed beam

\begin{tabular}{ccccccc}
\hline $\mathrm{m}=\mathrm{m}_{1} \times \mathrm{m}_{2}$ & $1 \times 1$ & $2 \times 1$ & $3 \times 1$ & $4 \times 1$ & $5 \times 1$ & $6 \times 1$ \\
\hline Even & 21.766 & 22.113 & 27.418 & 27.236 & 28.184 & 28.458 \\
compliance $(\mathrm{N} \cdot \mathrm{mm})$ & 3 & 2 & 1 & 6 & 5 & 7 \\
Total iteration steps & 52 & 44 & 52 & 59 & 52 & 44 \\
\hline
\end{tabular}

(2) two-dimensional sandwich structure

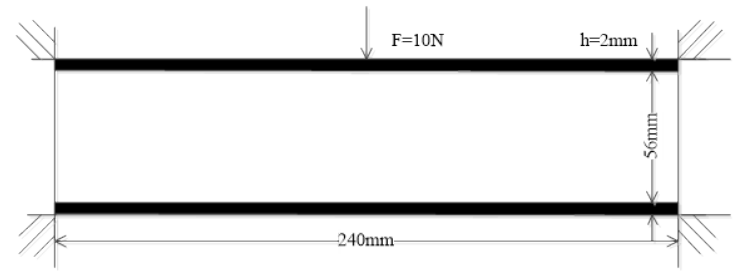

Fig. 4 Load and constraints of the two-dimensional sandwich structure

The load and constraints of the two-dimensional sandwich structure is shown in figure 4. The design area is $240 \mathrm{~mm} \times 56 \mathrm{~mm}$, and the non-design area is $240 \mathrm{~mm} \times 2 \mathrm{~mm}$. the design area is tied with 
the non-design area, and both of them have the same material properties. The sandwich structure is fixed at the both ends of skins. A vertical concentrated load $\mathrm{F}=10 \mathrm{~N}$ is applied at the center of the up face. The elastic modulus of structure is100MPa. Poisson's ratio is 0.3 . The volume constraint is 0.45 . Filter radius is $3 \mathrm{~mm}$. The design area is divided into different unit cells, and its final optimization structure is shown in the table 3 below.

Table 3 Optimal design for 2D sandwich structure under different constraints

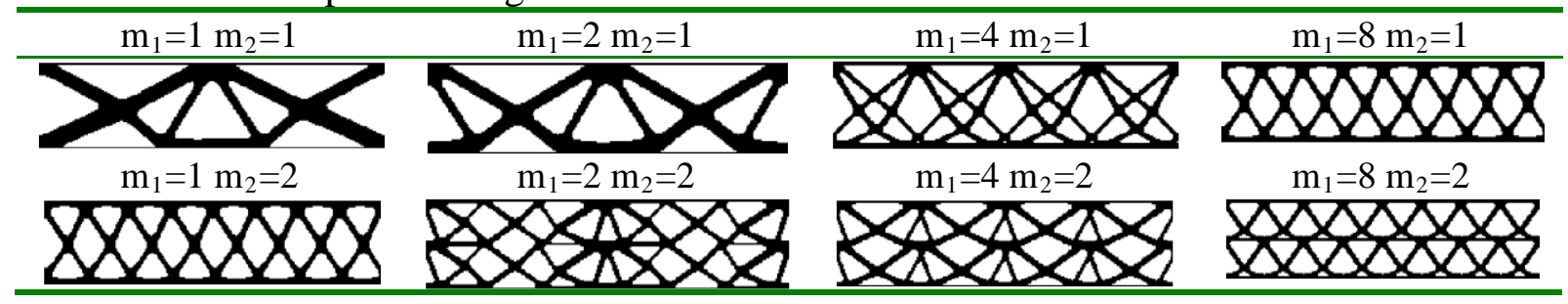

It can be seen from the above table 3, when the number of unit cell is equal, the divided way is different at the same time, the optimization structure has a obvious difference. The even compliance and the total iteration steps with the IGA-BESO method of the two-dimensional sandwich structure is shown in the following table.

Table 4 Even compliance and the total iteration steps of sandwich structure

\begin{tabular}{ccccccccc}
\hline $\mathrm{m}=\mathrm{m}_{1} \times \mathrm{m}_{2}$ & $1 \times 1$ & $2 \times 1$ & $4 \times 1$ & $8 \times 1$ & $1 \times 2$ & $2 \times 2$ & $4 \times 2$ & $8 \times 2$ \\
\hline $\begin{array}{c}\text { Even(N)mm) } \\
\text { compliance } \\
\begin{array}{c}\text { Total } \\
\text { iteration } \\
\text { steps }\end{array}\end{array}$ & 57.5976 & 74.3671 & 77.6122 & 92.2867 & 60.1098 & 77.7376 & 89.1814 & 99.1893 \\
\hline
\end{tabular}

It can be seen from table $4, m=m 1 \times m 2=1 \times 8$ and $m=m 1 \times m 2=2 \times 4$ have the same unit cell number, but their compliance have a big difference, this suggests that the latter form of cell division have more advantage to search the optimal solution.

\section{Summary}

It can get the following conclusion through the above analysis.

(1) IGA-BESO method can effectively conduct periodic structure topology optimization.

(2) The number of the unit cell and the divided way is different, the same structure have big difference in topology optimization.

(3) With the increase number of unit cell, the topology of the structure is more complicated and have higher compliance.

\section{References}

[1] X. Huang, Y. M. Xie. Evolutionary Topology Optimization of Continuum Structures: Methods and Applications. United Kingdom: John Wiley \& Sons Limited, 2010:111P.

[2] X. Huang, Y. M. Xie. Optimal design of periodic structures using evolutionary topology optimization. Structure Multidisc Optimization (2008) 36:597-606.

[3] Z. H. Zuo, Y. M. Xie, X. Huang. Combining Genetic Algorithms with BESO for Topology Optimization Algorithm. Applied Soft Computing, 2009, 9(4): 1343-1353. 